\title{
A REGIONAL L-BAND HIGH BIOMASS ESTIMATION FRAMEWORK LEVERAGING SPACEBORNE LIDAR AND INTERFEROMETRIC DATA TO OVERCOME BACKSCATTER SATURATION
}

\author{
Charlie Marshak $^{1}$, Marc Simard $^{1}$, Laura Duncanson $^{2,3}$, Carlos Silva ${ }^{2}$, Michael Denbina $^{1}$, Tien-Hao Liao $^{4}$ \\ ${ }^{1}$ Jet Propulsion Laboratory, California Institute of Technology, Pasadena, CA 91109, USA, \\ ${ }^{2}$ Department of Geographical Sciences, University of Maryland, College Park, MD 20742, USA \\ ${ }^{3}$ Biospheric Sciences Lab, NASA Goddard Space Flight Center, MD 20771, USA \\ ${ }^{4}$ Division of Geological and Planetary Sciences, California Institute of Technology, CA 91125, USA
}

\begin{abstract}
We propose a framework to estimate high above ground biomass (AGB) from L-band SAR imagery leveraging spaceborne lidars such as GEDI or ICESat-2 and repeat-pass coherence. Our results indicate we are able to overcome model saturation typically associated with purely backscatter methodologies. We validate our approach using lidar-derived AGB maps from the AfriSAR datasets at Mondah, Ogooue, and Lope. We apply our framework to UAVSAR and ALOS2 imagery to obtain 50 meter resolution biomass maps. We obtain $<60 \%$ nRMSE (in some cases much better) with negligible relative bias using a multiscale random forest model. We illustrate that the inclusion of coherence can significantly improve high AGB estimation particularly at the coastal site Mondah.
\end{abstract}

Index Terms - NISAR; GEDI; ICESat-2; L-band SAR; Microwave Remote Sensing; Biomass Estimation; AGB; data fusion.

\section{INTRODUCTION}

Spaceborne L-band SAR provides global coverage of Earth's above-ground biomass (AGB), which in turn can be used for environmental monitoring and carbon stock accounting [1]. However, inverting purely L-band $\mathrm{HH}+\mathrm{HV}$ models suffers from model saturation at approximately $100 \mathrm{Mg} / \mathrm{ha}[1,2]$. In this work, we overcome this difficulty by leveraging the sparse global sampling afforded with spaceborne lidar as in [3] as well as interferometric coherence. We propose training regional biomass models on these sparse but accurate lidar biomass estimates to create 50 meter biomass maps. We show that incorporating coherence and utilizing multiscale regression allows us to estimate tropical biomass at $<60 \%$ nRMSE $(<100 \mathrm{Mg} / \mathrm{ha}$ ) over the AfriSAR sites Mondah, Ogooue, and

This research was carried out at the Jet Propulsion Laboratory, California Institute of Technology, under a contract with the National Aeronautics and Space Administration. (C) 2019. California Institute of Technology. Government sponsorship acknowledged.
Lope. Further, we show that coherence provides a significant improvement in the coastal site of Mondah.

We are motivated in part by the extensive work to model forest structure with repeat-pass, short temporal baseline interferometric coherence $[4,5]$. We note that coherence is not used for biomass products for the upcoming NISAR mission, though such products will be available globally [1]. Indeed, environmental factors such as rain and even wind can impact temporal decorrelation observed in coherence images [1]. However, if model training is done regionally as we suggest here, the model can determine the correlative relationships between coherence and AGB on a site by site basis.

A comparison of this work to other biomass estimates is difficult in part because biomass data is fairly limited. We refer readers to the excellent review articles [2] and [6] for numerous AGB studies with radar remote sensing. We note that our reference data set provides orders of magnitude more biomass samples than those found in field measurement AGB studies as our reference data set is lidar-derived. We also note that radars with different frequencies and/or nonzero spatial baselines provide different insights into tree structure but may have caveats about availability. For example, the excellent results from [7] require quad-pol data, which are typically not available globally from spaceborne missions [1]. The strong results using Tandem-X such as [8] are valid only in low AGB forests such as in the boreal region, or require knowledge of the underlying ground topography. Our method does not have these limitations, because it uses the InSAR coherence magnitude but not the InSAR phase. The NISAR handbook specifies a $20 \mathrm{Mg} / \mathrm{ha} \mathrm{RMSE}$ for its biomass products, but only considers $80 \%$ of areas with biomass bounded by $100 \mathrm{Mg} / \mathrm{ha}$ [1]. Each site we consider in this work has mean biomass well above this $100 \mathrm{Mg} /$ ha threshold, with maximum biomass exceeding $600 \mathrm{Mg} / \mathrm{ha}$ across all sites, as indicated in Table 1. 


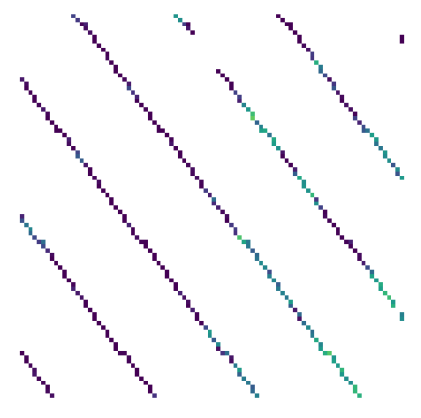

(a) Training Set

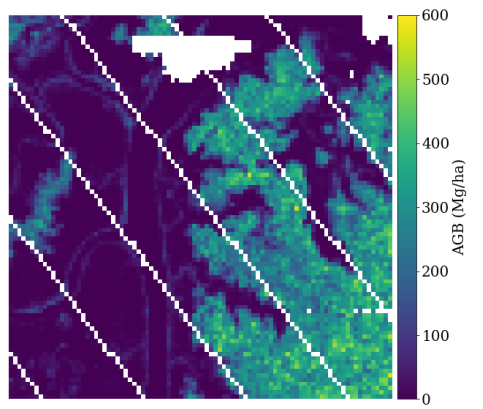

(b) Validation set

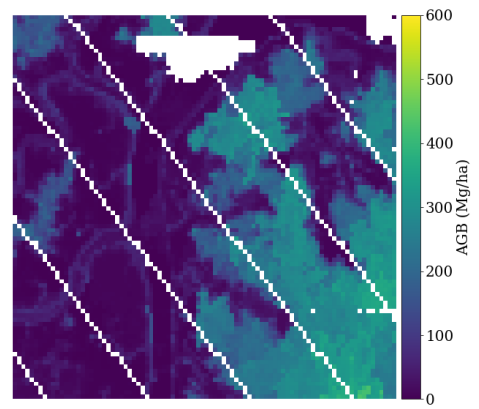

(c) Biomass estimates on validation set

Fig. 1: The train/validation framework to simulate GEDI spatial sampling from the lidar-derived AGB reference map.

\begin{tabular}{llccc}
\hline Sensor & Site & $\begin{array}{c}\mu_{\text {bio }} \\
(\mathrm{Mg} / \mathrm{ha})\end{array}$ & $\begin{array}{c}\sigma_{\text {bio }} \\
(\mathrm{Mg} / \mathrm{ha})\end{array}$ & $\begin{array}{c}\mu_{\text {bio }} / \sigma_{\text {bio }} \\
(\%)\end{array}$ \\
\hline ALOS-2 & Lope & 380.157 & 130.436 & 34.311 \\
& Mondah & 137.195 & 147.026 & 107.166 \\
& Ogooue & 286.896 & 123.461 & 43.033 \\
UAVSAR & Lope & 332.670 & 159.535 & 47.956 \\
& Mondah & 124.958 & 142.537 & 114.068 \\
& Ogooue & 256.626 & 137.885 & 53.730 \\
\hline
\end{tabular}

Table 1: Above, the mean and standard deviation of the biomass in the study areas with reference to the SAR sensor.

\section{DATASETS AND METHODOLOGY}

Our reference AGB map is lidar-derived. The map is created from methods described in [9] and will be used operationally for GEDI over tropical forests. For our SAR inputs, we utilize two L-band datasets to simulate NISAR capabilities: the airborne UAVSAR and the spaceborne ALOS-2. We obtain coherence images for each sensor using Kapok [10] and ISCE-2 [11] for UAVSAR and ALOS-2 respectively. The UAVSAR temporal baselines are a few hours, except for Ogooue where it is 8 days. The ALOS-2 pairs all have 14 day temporal baselines; longer temporal baselines are typically more difficult to extract forest structure from. We also obtain radiometrically and terrain corrected $\gamma^{0}$ backscatter images using the method found in [12]. We further despeckle using TV-MuLOG [13] without debiasing as our estimation model is invariant with respect to affine transformations of the inputs.

We then generate multiscale features for input into a random forest regression model [14]. Specifically, we segment the image using graph-based superpixels from [15] to generate a superpixel image pyramid as done in [16] extracting the mean and standard deviation of coherence and backscatter at each superpixel scale. The backscatter features are shown in Figure 2. Such multiscale features are becoming increasingly popular for forest estimations [17].
Our test-train framework is as follows. For training, we sample points spatially similar to that of GEDI on our reference AGB map and validate it on the remaining pixels. We generated training tracks along lines oriented 51.2 clockwise below the equator starting at the corner of the reference AGB map continuing to add tracks 120 meters eastward modulo the image extents until we return to or pass our first track. We did not include the orthogonal tracks but note that the more data for training used, the better our model performed. When reporting our results on the validation set, we consider pixels with at least $30 \%$ canopy cover as determined with [18]. This framework is illustrated in Figure 1.

\section{RESULTS}

Our validation set results are reported in Table 2 over areas with at least $30 \%$ canopy cover. Using this framework, we obtain $<60 \%$ nRMSE $(<100 \mathrm{Mg} / \mathrm{ha})$ on all sites and sensors used. The best nRMSE is at Lope at approximately $25 \%$ due in part to the homogeneity of the site's biomass (observe its low coefficient of variation in Table 1). Table 2 also compares the performance of a model trained with just backscatter and another trained with both backscatter and coherence. Over the coastal site Mondah, we obtain significant improvements of our biomass estimates with each sensor when we include coherence. Note that across all site and sensors the inclusion of coherence improves our estimates. Figure 3 shows the Mondah site biomass reference and biomass estimates from our model using UAVSAR data. The model captures the spatial structure of the AGB throughout the area.

\section{CONCLUSIONS}

We illustrate that we can estimate AGB in high biomass areas leveraging sparse lidar measurements and interferometric coherence in order to overcome model saturation typically observed when estimating AGB from SAR backscatter using traditional models. Our comparisons show that coherence 


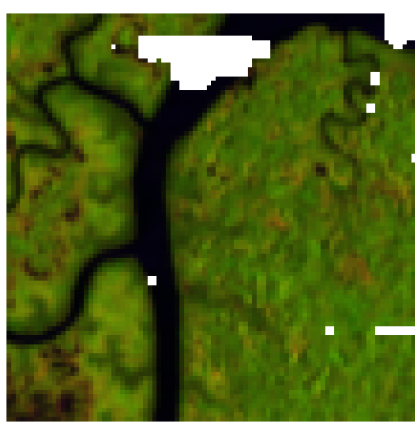

(a)

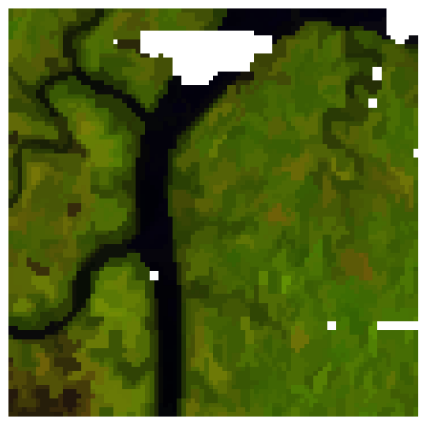

(b)

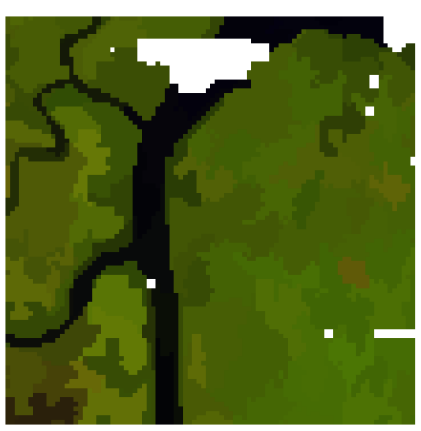

(c)

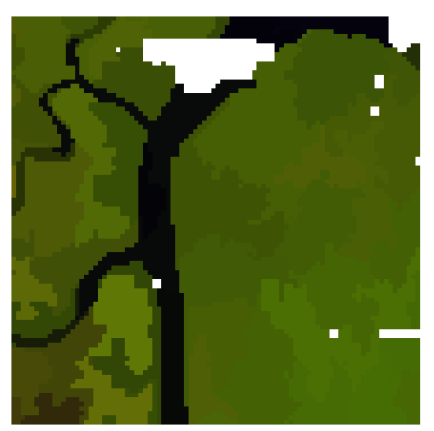

(d)

Fig. 2: Backscatter features derived from graph-based superpixels [15] with increasing minimum size. From left to right, (A) original pixel data projected to biomass frame (50 meters resolution), (B) mean backscatter with minimum segment size 5 (.025 ha), (C) mean backscatter with minimum segment size 25 pixels (.125 ha), (D) mean backscatter with minimum segment size 50 pixels (.25 ha). Red is $\mathrm{HH}$, Green is $\mathrm{HV}$, and Blue is $\mathrm{HH} / \mathrm{HV}$.

\begin{tabular}{|c|c|c|c|c|}
\hline Site & Products & $\begin{array}{l}\text { RMSE } \\
(\mathrm{Mg} / \mathrm{ha})\end{array}$ & $\begin{array}{c}\text { nRMSE } \\
(\%)\end{array}$ & $\begin{array}{c}\text { Bias } \\
(\mathrm{Mg} / \mathrm{ha})\end{array}$ \\
\hline \multicolumn{5}{|c|}{ ALOS-2 } \\
\hline \multirow[t]{2}{*}{ Mondah } & Backscatter & 84.20 & 61.94 & -1.26 \\
\hline & $\begin{array}{l}\text { Backscatter and } \\
\text { Coherence }\end{array}$ & 72.54 & 53.67 & -2.03 \\
\hline \multirow[t]{2}{*}{ Lope } & Backscatter & 99.70 & 26.29 & -0.87 \\
\hline & $\begin{array}{l}\text { Backscatter and } \\
\text { Coherence }\end{array}$ & 96.46 & 25.43 & -0.82 \\
\hline \multirow[t]{2}{*}{ Ogooue } & Backscatter & 99.63 & 34.38 & 2.93 \\
\hline & $\begin{array}{l}\text { Backscatter and } \\
\text { Coherence }\end{array}$ & 96.69 & 33.43 & 2.33 \\
\hline \multicolumn{5}{|c|}{ UAVSAR } \\
\hline \multirow[t]{2}{*}{ Mondah } & Backscatter & 81.79 & 64.12 & 2.60 \\
\hline & $\begin{array}{l}\text { Backscatter and } \\
\text { Coherence }\end{array}$ & 72.80 & 56.75 & 3.34 \\
\hline \multirow[t]{2}{*}{ Lope } & Backscatter & 84.14 & 25.14 & 1.98 \\
\hline & $\begin{array}{l}\text { Backscatter and } \\
\text { Coherence }\end{array}$ & 82.19 & 24.46 & 3.31 \\
\hline \multirow{2}{*}{$\begin{array}{l}\text { Ogooue } \\
\text { (Rain) }\end{array}$} & Backscatter & 97.62 & 38.32 & -1.90 \\
\hline & $\begin{array}{l}\text { Backscatter and } \\
\text { Coherence }\end{array}$ & 92.33 & 36.17 & -1.35 \\
\hline
\end{tabular}

Table 2: Above we use the model with all superpixel features and compare the difference when the model uses backscatter and when the model uses both backscatter and coherence.

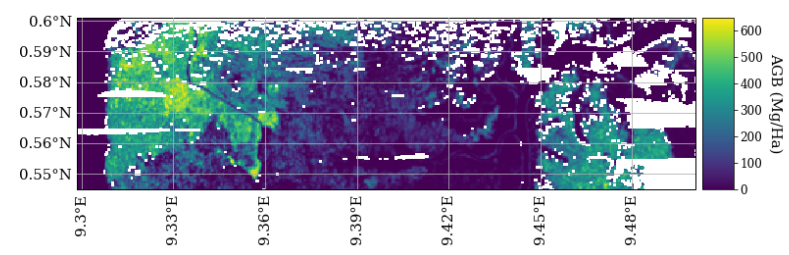

(a) Biomass Reference

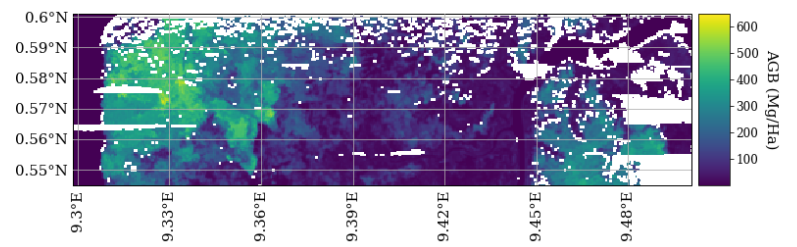

(b) Biomass Estimates

Fig. 3: Comparing the biomass reference map (top) with those derived from our model over Mondah with UAVSAR.

in particular can provide significant improvements to high biomass estimates compared to biomass estimates generated from SAR backscatter alone. Our multiscale model is able to capture spatial correlations and leverage such correlations into high AGB estimates. We hope that this approach and study will be valuable for future biomass products associated with NISAR. In future work, we will explore uncertainties and model parameters further. Additionally, we will apply this estimation framework to boreal forests.

\section{REFERENCES}

[1] NISAR Science Team, "NASA-ISRO SAR Mission Science Users Handbook," https://nisar.jpl.nasa.gov/files/ nisar/NISAR_Science_Users_Handbook.pdf, 2019, [Online; accessed January-2019]. 
[2] Sinha, S. and Jeganathan, C. and Sharma, L. K. and Nathawat, M. S., "A review of radar remote sensing for biomass estimation," International Journal of Environmental Science and Technology, vol. 12, no. 5, pp. 1779-1792, 2015.

[3] L. Duncanson, A. Neuenschwander, S. Hancock, N. Thomas, T. Fatoyinbo, M. Simard, S. Luthcke, C. Silva, J. Armston, M. Hofton, and R. Dubayah, "Understanding biomass errors from simulated gedi, icesat-2 and nisar data across environmental gradients in sonoma county, california," Remote Sensing of Environment, submitted.

[4] M. Lavalle, M. Simard, and S. Hensley, "A Temporal Decorrelation Model for Polarimetric Radar Interferometers," IEEE Transactions on Geoscience and Remote Sensing, vol. 50, no. 7, pp. 2880-2888, 2011.

[5] M. Simard, S. Hensley, M. Lavalle, R. Dubayah, N. Pinto, and M. Hofton, "An Empirical Assessment of Temporal Decorrelation using the Uninhabited Aerial Vehicle Synthetic Aperture Radar over Forested Landscapes," Remote Sensing, vol. 4, no. 4, pp. 975-986, 2012.

[6] M. Stelmaszczuk-Górska, C. Thiel, and C. Schmullius, "Remote Sensing for Aboveground Biomass Estimation in Boreal Forests," Earth Observation for Land and Emergency Monitoring..; Balzter, H., Ed, pp. 33-55, 2017.

[7] M. Neumann, S. S. Saatchi, L. M. H. Ulander, and J. E. S. Fransson, "Assessing Performance of Land P-band Polarimetric Interferometric SAR Data in Estimating Boreal Forest Above-Ground Biomass," IEEE Transactions on Geoscience and Remote Sensing, vol. 50, no. 3, pp. 714-726, 2012.

[8] J. Askne, J. Fransson, M. Santoro, M. Soja, and L. Ulander, "Model-based Biomass Estimation of a Hemiboreal Forest from Multitemporal TanDEM-X Acquisitions," Remote Sensing, vol. 5, no. 11, pp. 5574-5597, 2013.

[9] W. Huang, A. Swatantran, L. Duncanson, K. Johnson, D. Watkinson, K. Dolan, J. O'Neil-Dunne, G. Hurtt, and R. Dubayah, "County-Scale Biomass Map Comparison: A Case Study for Sonoma, California," Carbon Management, vol. 8, no. 5-6, pp. 417-434, 2017.

[10] M. Denbina and M. Simard, "Kapok: An Open Source Python Library for PolInSAR Forest Height Estimation using UAVSAR Data," in 2017 IEEE International Geoscience and Remote Sensing Symposium (IGARSS). IEEE, 2017, pp. 4314-4317.
[11] P. Rosen, E. Gurrola, P. S. Agram, G. F. Sacco, and M. Lavalle, "The InSAR Scientific Computing Environment (ISCE): A Python Framework for Earth Science," in AGU Fall Meeting Abstracts, 2015.

[12] M. Simard, B. V. Riel, M. Denbina, and S. Hensley, "Radiometric Correction of Airborne Radar Images over Forested Terrain with Topography," IEEE Transactions on Geoscience and Remote Sensing, vol. 54, no. 8, pp. 4488-4500, 2016.

[13] C.-A. Deledalle, L. Denis, S. Tabti, and F. Tupin, "MuLoG, or How to Apply Gaussian Denoisers to multichannel SAR Speckle Reduction?" IEEE Transactions on Image Processing, vol. 26, no. 9, pp. 4389-4403, 2017.

[14] L. Breiman, "Random forests," Machine learning, vol. 45, no. 1, pp. 5-32, 2001.

[15] P. F. Felzenszwalb and D. P. Huttenlocher, "Efficient Graph-based Image Segmentation," International Journal of Computer Vision, vol. 59, no. 2, pp. 167-181, 2004.

[16] B. Bonev and A. L. Yuille, "Bottom-Up Processing in Complex Scenes: A Unifying Perspective on Segmentation, Fixation Saliency, Candidate Regions, BaseDetail Decomposition, and Image Enhancement," in Recent Progress in Brain and Cognitive Engineering. Springer, 2015, pp. 109-130.

[17] N. Lang, K. Schindler, and J. D. Wegner, "CountryWide High-Resolution Vegetation Height Mapping with Sentinel-2," Remote Sensing of Environment, vol. 233, p. 111347, 2019. [Online]. Available: http://www.sciencedirect.com/science/article/pii/ S0034425719303669

[18] M. C. Hansen, P. V. Potapov, R. Moore, M. Hancher, S. A. A. Turubanova, A. Tyukavina, D. Thau, S. V. Stehman, S. J. Goetz, T. R. Loveland et al., "Highresolution Global Maps of 21st Century Forest Cover Change," Science, vol. 342 , no. 6160, pp. 850-853, 2013. 